\title{
A PROOF OF THE RIEMANN HYPOTHESIS Jamel Ghanouchi
}

RIME department of Mathematics

\section{Abstract}

In this paper, we present the Riemann problem and define the real primes. It allows to generalize the Riemann hypothesis to the reals. A calculus of integral solves the problem. We generalize the proof to the integers.

\section{The Riemann hypothesis}

The Riemann conjecture is a conjecture which has been formulated in 1859 by the subjet of the Riemann funtion zeta or $\zeta$. It is called the zeta Riemann func $n$.

This function is defined as follows

$$
\zeta(s)=\sum_{n=1}^{n=\infty}\left(\frac{1}{n^{s}}\right)=1+\frac{1}{2^{s}}+\frac{1}{3^{s}}+
$$

The first result is the divergence of the harmonic serie

$$
\zeta(1)=\sum_{n=1}^{n=\infty}\left(\frac{1}{-1}\right)=1+\frac{1}{2}
$$

It has been proved in the middle age by Nicole $d$ esn

In the XVIII century, Leonard Euler has discover the main proprieties of the $\zeta$ function.

In the 1730's he conjectured afte umer cal calcu as the following equality, which is often called the Basel problem. 
With Bernard Riemann, s can be complex number. Riemann proved the following formula

Where

$$
\pi^{\frac{-s}{2}} \Gamma\left(\frac{s}{2}\right) \zeta(s)=\pi^{\frac{-(1-s)}{2}} \Gamma\left(\frac{1-s}{2}\right) \zeta(1-s)
$$

$$
\Gamma(s)=\int_{0}^{\infty} t^{s-1} e^{-t} d t
$$

This formula demonstrates that this equation does not change if we replace $\mathrm{s}$ by 1 -s.

Thus it is symmetric $\mid s=\frac{1}{2}$

Riemann demonstrates that the only zeros in the $R(s)<0$ are the trivial zero negativen numbers and that there is no zero in the $R(s)>1$.

The other zeros are the non trivial zeros. They are in the critical zone

Riemann conjectured theu are all in the critical line $R(s)=\frac{1}{2}$.

This conjecture is called the Riemann hypothesis.

They calculated numerically one billion zeros of the Rieman y are located in the critical line.

\section{Definition}

A real number is compound if it can be written as

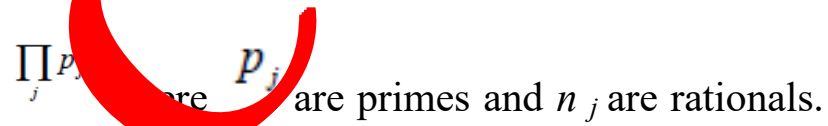

This decomposition in prime factors is unique rime rearnumber can be written only as $\mathrm{p}=\mathrm{p} .1$.

Thus we define other real prime numbers like $\pi / \mathrm{e}, 1(2)$. Thus $\sqrt[q]{p}=p^{\frac{1}{q}}$ is compound. Also $\sqrt[q]{p}+1=p^{\frac{1}{q}}$ is prime when $\mathrm{p}$ is $y$ ne an we har $\sqrt[z]{p}-1=(p-1)(\sqrt[2 i]{p}+1)^{-1}\left(2^{2-1} \sqrt{p}+1\right)^{-1} \ldots(\sqrt{p}+1)^{-1}$ compound for $\mathrm{p}$ prime, for exa ple.

\section{The approach of the $R^{\circ}$ mapothes}

The Rieman hyp chesis tates that e non trivial zeros of the Riemann zeta function $\zeta(z)=\sum_{t=1}^{\infty} \frac{1}{t^{z}}$ lie on the critical line

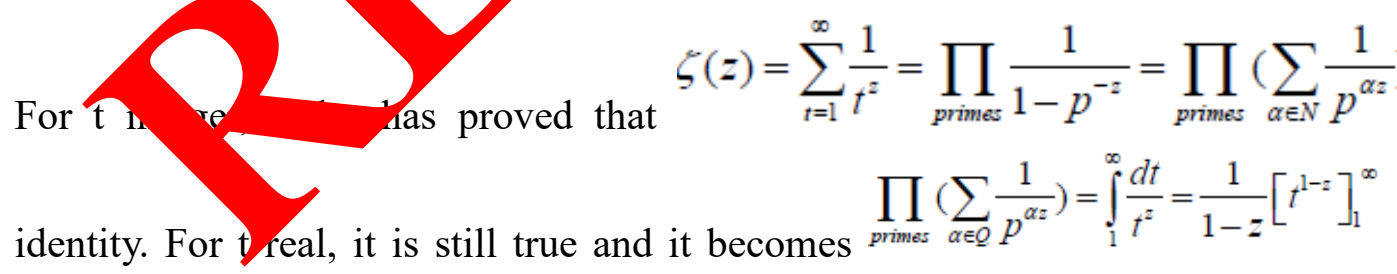

tivial zeros : we have $\zeta(-2 k)=0, \forall k \in N$ and $\left[t^{1+2 k}\right]_{1}^{\infty}=0$ but if $\left[t^{z}\right]^{\infty}$ s the limit in the infinity,

$$
\begin{aligned}
& \lim _{t \rightarrow \infty}\left(t^{1+2 k}\right)=1, \forall k \in N \text { and } \\
& \lim _{i \rightarrow \infty}\left(t^{1-\frac{1}{2}-i y}\right)=\lim _{i \rightarrow \infty}\left(t^{\frac{1}{2}-i y}\right)=\lim _{i \rightarrow \infty}\left(t^{\frac{1}{2}+i y}\right)=\frac{1}{2}\left(\lim _{i \rightarrow \infty}\left(t^{\frac{1}{2}-i y}\right)+\lim _{i \rightarrow \infty}\left(t^{\frac{1}{2}+i y}\right)\right)=a \\
& \Rightarrow \lim _{i \rightarrow \infty}\left(t^{2\left(\frac{1}{2}+i y\right)}-2 a t^{\frac{1}{2}+i y}+t\right)=\lim _{i \rightarrow \infty}\left(t^{2\left(\frac{1}{2}+i y\right)}-2 a t^{\frac{1}{2}+i y}+1\right)=0 \Rightarrow \lim _{i \rightarrow \infty}\left(t^{\frac{1}{2}+i y}\right)=a=a+\sqrt{a^{2}-1}=1
\end{aligned}
$$


it means that $\zeta\left(\frac{1}{2}+i y\right)=\left[\frac{1}{1-\frac{1}{2}-i y} t^{1-\frac{1}{2}-i y}\right]_{1}^{\infty}=0$

Let now $\zeta(x+i y)=0=\left[\frac{t^{1-x-i y}}{1-x-i y}\right]_{1}^{\infty}=\left[\frac{t^{\frac{1}{2}-i y} t^{\frac{1}{2}-x}}{1-x-i y}\right]_{1}^{\infty}=\left[\frac{t^{\frac{1}{2}-x}}{1-x-i y}\right]_{1}^{\infty}=0 \Rightarrow x=\frac{1}{2}$

We have proved that the non trivial zeros of the Riemann function for the reals lie in the critical line ! So the hypothesis is proved for the real numbers. The Riemann hypothesis is important because it gives information about the zeros of the Riemann function and the distribution of those zeros are related to real primes!

\section{The generalization to the integers}

We have

$$
\begin{aligned}
& \int_{1}^{\infty} \frac{d t}{t^{z}}=\frac{1}{1-z}\left[t^{1-z}\right]_{1}^{\infty}=\sum_{t=1}^{\infty} \frac{1}{t^{z}}+\sum_{\alpha \in Q^{-}}^{\infty} \frac{1}{t^{\prime \alpha z}}+\sum_{\alpha \in Q^{+} \backslash N}^{\infty} \frac{1}{t^{\prime \alpha z}} \\
& =\sum_{t=1}^{\infty} \frac{1}{t^{z}} \cdot B=\prod_{\text {primes }}\left(\sum_{\alpha \in N} \frac{1}{p^{\alpha z}}\right) \cdot B=\sum_{t=1}^{\infty} \frac{1}{t^{z}}+A
\end{aligned}
$$

Let now

$$
\prod_{\text {primes }}\left(\sum_{\alpha \in N} \frac{1}{p^{\alpha(x+i y)}}\right)=0
$$

We have : If $\mathrm{B}$ is finite, the zeta function for $\mathrm{e}$ cals $\mathrm{M}$ be equal to zero. Else Let $\zeta_{1}(\mathrm{z})$ the Riemann function for the reals and $\zeta$ he Riem nn function fot the integers, if $\mathrm{B}=+\infty$ and if $\mathrm{A}$ is finite and

A $>1$

$$
\begin{aligned}
& (A+\zeta(z))^{2}=A^{2}=A^{2},(z)^{2}+2 \lambda(z)=A^{2}+2 A \zeta(z) \Rightarrow A \zeta(z)=0 \\
& \left(A^{n} \zeta(z)+A\right)^{2}=A^{2}=\left(A^{2}+{ }^{n} \zeta(z)\right)^{2}+2 A^{n+1} \zeta(z) \Rightarrow A^{n+1} \zeta(z)=0, \forall n \\
& \exists \alpha \mid B \quad A^{\alpha}<\Rightarrow \beta<\zeta_{1}(z), B \zeta(z)<A^{n} \zeta(z)=0 \Rightarrow \zeta_{1}(z)=0 \Rightarrow 1 \geq A \\
& \text { And if } \\
& 1 \geq A \\
& \left(A^{-2}(z)-A^{-2}\right)=A^{-2}+\zeta(z)^{2}+2 A^{-1} \zeta(z)=A^{-2}+2 A^{-1} \zeta(z) \Rightarrow A^{-1} \zeta(z)=0 \\
& \left(A^{-n} \zeta(z) 1^{-1}\right)^{2}=A^{-2}=A^{-2}+\left(A^{-n} \zeta(z)\right)^{2}+2 A^{-n-1} \zeta(z) \Rightarrow A^{-n-1} \zeta(z)=0, \forall n \\
& \exists \alpha \mid B \quad A^{-\alpha}<A^{-n} \Rightarrow 0<\zeta_{1}(z)=B \zeta(z)<A^{-n} \zeta(z)=0 \Rightarrow \zeta_{1}(z)=0
\end{aligned}
$$$$
0 \geq A \geq-1
$$$$
\left(A^{-1}+\zeta(z)\right)^{2}=A^{-2}=A^{-2}+\zeta(z)^{2}+2 A^{-1} \zeta(z)=A^{-2}+2 A^{-1} \zeta(z) \Rightarrow A^{-1} \zeta(z)=0
$$$$
\left(A^{-2 n-1} \zeta(z)+A^{-2}\right)^{2}=A^{-4}=A^{-4}+\left(A^{-2 n-1} \zeta(z)\right)^{2}+2 A^{-2 n-3} \zeta(z) \Rightarrow A^{-2 n-3} \zeta(z)=0, \forall n
$$$$
\exists \alpha \mid B \quad-A^{-\alpha}<-A^{-2 n-1} \Rightarrow 0<\zeta_{1}(z)=B \zeta(z)<-A^{-2 n-1} \zeta(z)=0 \Rightarrow \zeta_{1}(z)=0
$$ 
And if

$A<-1$

$(A+\zeta(z))^{2}=A^{2}=A^{2}+\zeta(z)^{2}+2 A \zeta(z)=A^{2}+2 A \zeta(z) \Rightarrow A \zeta(z)=0$

$\left(A^{2 n+1} \zeta(z)+A^{2}\right)^{2}=A^{4}=A^{4}+\left(A^{2 n+1} \zeta(z)\right)^{2}+2 A^{2 n+3} \zeta(z) \Rightarrow A^{2 n+3} \zeta(z)=0, \forall n$

$\exists \alpha \mid B-A^{\alpha}<-A^{2 n+1} \Rightarrow 0<\zeta_{1}(z)=B \zeta(z)<A^{2 n+1} \zeta(z)=0 \Rightarrow \zeta_{1}(z)=0 \Rightarrow A \geq-1$

If now $\mathrm{B}=-\infty$ and if $\mathrm{A}$ is finite and

$A>1$

$(A+\zeta(z))^{2}=A^{2}=A^{2}+\zeta(z)^{2}+2 A \zeta(z)=A^{2}+2 A \zeta(z) \Rightarrow A \zeta(z)=0$

$\left(A^{n} \zeta(z)+A\right)^{2}=A^{2}=A^{2}+\left(A^{n} \zeta(z)\right)^{2}+2 A^{n+1} \zeta(z) \Rightarrow A^{n+1} \zeta(z)=, \nabla \eta$

$\exists \alpha \mid B \quad-A^{\alpha}>-A^{n} \Rightarrow 0>\zeta_{1}(z)=B \zeta(z)>-A^{n} \zeta(z)=0 \Rightarrow>1$

And if

$1 \geq A \geq 0$

$\left(A^{-1}+\zeta(z)\right)^{2}=A^{-2}=A^{-2}+\zeta(z)^{2}+2 A^{-1} \zeta(z)=4^{-2}+2 A^{-1} \zeta(z) \Rightarrow A^{-1} \zeta(z)=0$

$\left(A^{-n} \zeta(z)+A^{-1}\right)^{2}=A^{-2}=A^{-2}+\left(A^{-n} \zeta(z)\right)^{2}+2 A \quad \zeta(z)=A^{-n-1} \zeta(z)=0, \forall n$

$\exists \alpha \mid B \quad-A^{-\alpha}>-A^{-n} \Rightarrow 0>\zeta_{1}(z)=B \zeta \Rightarrow A^{-n} \zeta(z)=0 \Rightarrow \zeta_{1}(z)=0$

And if

$0 \geq A \geq-1$

$\left(A^{-1}+\zeta(z)\right)^{2}=A^{-2}=4(z)^{2} A^{-1} \zeta(z)=A^{-2}+2 A^{-1} \zeta(z) \Rightarrow A^{-1} \zeta(z)=0$

$\left(A^{-2 n-1} \zeta(z)+A^{-2} A^{-4}=-4+\left(A^{-2 n-1} \zeta(z)\right)^{2}+2 A^{-2 n-3} \zeta(z) \Rightarrow A^{-2 n-3} \zeta(z)=0, \forall n\right.$

$\exists \alpha \mid B \quad A^{-\alpha}<A^{-2 n} \Rightarrow \rho>\zeta_{1}(z)=B \zeta(z)>A^{-2 n-1} \zeta(z)=0 \Rightarrow \zeta_{1}(z)=0$

And if

$A<$

$\left(A+\zeta(z), A^{2}=A^{2}+\zeta(z)^{2}+2 A \zeta(z)=A^{2}+2 A \zeta(z) \Rightarrow A \zeta(z)=0\right.$

$\left(A^{2 n+1} \zeta(z)+A^{2}\right)^{2}=A^{4}=A^{4}+\left(A^{2 n+1} \zeta(z)\right)^{2}+2 A^{2 n+3} \zeta(z) \Rightarrow A^{2 n+3} \zeta(z)=0, \forall n$

$\exists \alpha \mid B \quad A^{\alpha}>A^{2 n+1} \Rightarrow 0>\zeta_{1}(z)=B \zeta(z)>A^{2 n+1} \zeta(z)=0 \Rightarrow \zeta_{1}(z)=0 \Rightarrow A \geq-1$

And if $A= \pm \infty$ and we have $B \mid A^{\alpha+1}$ hence

$A^{\alpha} \zeta(z)^{2}=\frac{A^{\alpha+2}}{\left(A^{\alpha+1}-1\right)^{2}}=0$

$A^{\alpha} \zeta(z)=1$ 
And

$\left(A^{\alpha} \zeta(z)+A^{\alpha} \zeta(z)^{2}\right)^{2}=A^{2 \alpha} \zeta(z)^{2}=A^{2 \alpha} \zeta(z)^{2}+2 A^{2 \alpha} \zeta(z)^{3} \Rightarrow A^{2 \alpha} \zeta(z)^{3}=0$

If $A^{n \alpha} \zeta(z)^{n+1}=0$ then

$\left(A^{n \alpha} \zeta(z)^{n+1}+A^{\alpha} \zeta(z)\right)=A^{2 \alpha} \zeta(z)^{2}=A^{2 \alpha} \zeta(z)^{2}+2 A^{(n+1) \alpha} \zeta(z)^{n+2} \Rightarrow A^{(n+1) \alpha} \zeta(z)^{n+2}=0, \forall n$

$\Rightarrow A^{\alpha} \zeta(z)^{\frac{n+1}{n}}=0, \forall n \Rightarrow A^{\alpha} \zeta(z)=0=\frac{A^{\alpha+1}}{A^{\alpha+1}-1}=1$

It is impossible !

$\Rightarrow A \neq \infty, A \neq-\infty$

Thus $\mathrm{A}=0$ And

$\int_{1}^{\infty} \frac{d t}{t^{x+i y}}=\frac{1}{1-x-i y}\left[t^{1-x-i y}\right]_{1}^{\infty}=\prod_{p r i m e s}\left(\sum_{\alpha \in N} \frac{1}{p^{\alpha(x+i y)}}\right) \cdot B=\prod_{p \text { primes }}\left(\sum_{\alpha \in N} \frac{1}{p^{\alpha(x+i y)}}\right)+$

Thus the non trivial zeros of the Riemann funtion zeta lie in the the proof of the Riemann hypothesis !

\section{Conclusion}

We have generalized the concept of prime to the rea It allowed to prove the conjecture to the reals. Then, we have proved the Riemann hypothesis.

\section{The Bibliography}

[1] R. J. Backlund, "Sur les zéros de la foncti ל(s) Ce Riemann », CRAS, vol. 158, 1914, p. 1979-1981.

[2] X. Gourdon, «The 1013 first large height » [3] J.P.Gram, « Note su k Ǵros de là Anction $\zeta($ s) de Riemann », Acta Mathematica, vol. 27,
1903, p. 289-304.

[4] J.I.hutchinson On the Roots y Ae Riemann Zeta-Function », Trans. AMS, vol. 27, no 1, 1925, p. 49-60.

[5] A. M. Odlyzko, e 1020 ch zero of the Riemann zeta function and 175 million of its neighbors, 1992.

[6] V. Jey Losser. J/M. Yohe et Lowell Schoenfeld, « Rigorous computation and the zeros of the Riemann q-Iumuon.», Information Processing 68 (Proc. IFIP Congress, Edinburgh, 1968), Vol. 1: Mathemâ Software, Amsterdam, North-Holland, 1969, p. 70-76.

[7] E.C.Titchnarsh, «The Zeros of the Riemann Zeta-Function », Proceedings of the Royal Society, Series A, Mathematical and Physical Sciences, vol. 151, no 873, 1935, p. 234-255.

[8] E. C. Titchmarsh, "The Zeros of the Riemann Zeta-Function », Proceedings of the Royal Society, Series A, Mathematical and Physical Sciences, The Royal Society, vol. 157, no 891, 1936, p. 261-263.

[9] A.M.Turing, "Some calculations of the Riemann zeta-function », Proceedings of the LMS, Third Series, vol. 3, 1953, p. 99-117.

[10] J. van de Lune, H.te Riele et D. T. Winter, « On the zeros of the Riemann zeta function in the critical strip. IV », Mathematics of Computation, vol. 46, no 174, 1986, p. 667-681. 